\title{
Middle Ear Dysfunction: Connection to Allergic Rhinitis
}

\author{
${ }^{1}$ Shilpam Sharma, ${ }^{2}$ Abhinav Srivastava, ${ }^{3}$ Chander Mohan Sharma
}

\begin{abstract}
Introduction: The correlation between middle ear pathology due to Eustachian tube dysfunction and nasal allergy has been a topic of debate since long. Studies of the pathogenesis of otitis media have identified interactions among infection, allergic reactions and Eustachian tube dysfunction. AIMS- The aim of this study was to evaluate the relationship between Eustachian tube dysfunction and nasal allergy and to evaluate the efficacy of oral steroids and intranasal steroidal spray on Eustachian tube dysfunction.
\end{abstract}

Materials and methods: The patients were diagnosed according to the diagnostic criteria of allergic rhinitis. A total of 50 patients having moderate/severe symptoms who had not taken any treatment for allergic rhinitis in the past 6 weeks with deranged middle ear function were included in the study. Group I patients were given Prednisolone, $30 \mathrm{mg}$ once daily for 10 days in tapering dose along with intranasal steroid spray for 6 weeks. Group II patients were given only intranasal steroid spray for 6 weeks. Impedance audiometry was repeated at the end of 6 weeks and results were statistically analyzed.

Result: This study demonstrated the outcome of systemic steroids and intranasal steroidal spray in the treatment of middle ear dysfunction. In our study impedance audiometry was done at the start of study and showed type B tympanogram in $36 \%$ ears in group I and $21 \%$ in group II and type $C$ tympanogram in $60 \%$ in group I and $50 \%$ in group II. Impedance audiometry was repeated at 10 days of treatment and showed reversal to type A curve in $26 \%$ of ears in group I and $14 \%$ of ears in group II. Impedance audiometry at the end of treatment demonstrated reversal to type A tympanogram in $64 \%$ of ears in group I and $48 \%$ of ears in group II.

Keywords: Allergic rhinitis, Intranasal steroidal spray, Middle ear dysfunction, Oral steroids.

How to cite this article: Sharma S, Srivastava A, Sharma CM. Middle Ear Dysfunction: Connection to Allergic Rhinitis. Clin Rhinol An Int J 2016;9(2):65-67.

Source of support: Nil

Conflict of interest: None

\section{INTRODUCTION}

Function of Eustachian tube is ventilation and clearance of middle ear as well as mechanical and immunological

\footnotetext{
${ }^{1}$ Junior Resident, ${ }^{2}$ Assistant Professor, ${ }^{3}$ Professor and Head

${ }^{1-3}$ Department of ENT and Head and Neck Surgery, Rohilkhand Medical College, Bareilly, Uttar Pradesh, India

Corresponding Author: Shilpam Sharma, Junior Resident Department of ENT and Head and Neck Surgery, Rohilkhand Medical College, Bareilly, Uttar Pradesh, India, Phone: +919760612907, e-mail: shilpam30@gmail.com
}

defense. Common etiological factors for Eustachian tube dysfunction include infections, obstructive causes, genetics, reflux diseases, allergy, and iatrogenic causes.

Eustachian tube dysfunction can be due to mechanical or functional obstruction leading to acute or chronic otitis media with effusion. In experiments, allergic rhinitis provoked in patients with a history of allergy has been associated with the development of Eustachian tube obstruction. ${ }^{1}$ Studies in the pathogenesis of otitis media have found that atopic children are more prone to develop bilateral OME, to present with type B tympanogram and have a lower threshold of hearing as compared to nonatopic children. ${ }^{2,3}$

\section{AIM}

The aim of this study was to evaluate the relationship between Eustachian tube dysfunction and nasal allergy and to evaluate the efficacy of oral steroids and intranasal steroidal spray on Eustachian tube dysfunction.

\section{MATERIALS AND METHODS}

A prospective clinical study was carried out from June 2014 to May 2015 on 50 patients who were evaluated in the Department of Otolaryngology of a tertiary care hospital and had been diagnosed to have deranged middle ear function. The age of the patients ranged between 15 and 50 years. All the patients diagnosed to have been suffering from moderate/severe symptoms of allergic rhinitis (one or more symptoms - sleep disturbance, impairment of daily activities, sport, leisure, impairment of school or work, troublesome symptoms) with deranged middle ear function and had not taken any treatment for allergic rhinitis were enrolled for the study. Patients were divided into 2 groups of 25 patients each. Patients in group I were given prednisolone $30 \mathrm{mg}$ once daily for 10 days in tapering dose along with intranasal steroid spray for 6 weeks. Group II patients were given only intranasal steroid spray for 6 weeks. Impedance audiometry was repeated at the end of 6 weeks and results were statistically analyzed. The following patients were excluded from the study as follows:

- Sensorineural hearing loss

- History of immunodeficiency

- Hypersensitivity to fluticasone nasal spray

- Systemic and local contraindication against steroids. 


\section{RESULT}

This study has been carried out in a tertiary care teaching hospital in western Uttar Pradesh, India. A total of 50 patients were taken up for the study (Table 1). There were 22 males and 28 females. All the patients were selected as per the decided criteria. All patients had moderate and severe symptoms of allergic rhinitis and they were off treatment for allergic rhinitis for the past 6 weeks. Patients were evaluated on their first visit by impedance audiometry and patients were then divided into 2 groups. Group I patients were given oral as well as intranasal steroids and group II patients were given only intranasal steroids. Oral steroids were given for a period of 10 days and intranasal steroids were given for 6 weeks in patients of both the groups. All the patients underwent impedance audiometry before start of study, after 10 days and after 6 weeks (Tables 2-4, Graphs 1 and $2)$. The results were tabulated and statistically analyzed.

- The impedance audiogram, in group I, “ $\mathrm{B}^{\prime}$ c curve was demonstrated in 18 out of 50 ears (36\%). Among these, 4 became " $\mathrm{A}$ " type after 10 days of treatment and total 14 ear tympanogram converted into A type at the end of treatment.

- " $\mathrm{C}$ " curve was seen in 30 out of 50 ears (60\%). Among these, 9 became A type after 10 days of treatment and

Table 1: Age and sex distribution among groups I and II

\begin{tabular}{lll}
\hline Age distribution & Male & Female \\
\hline $15-25$ years & 7 & 15 \\
$26-36$ years & 10 & 7 \\
$37-50$ years & 5 & 6 \\
\hline
\end{tabular}

Table 3: Impedance audiometry 10 days after treatment

\begin{tabular}{lll}
\hline Tympanogram & Group I & Group II \\
\hline Type B & $14(28 \%)$ & $19(38 \%)$ \\
Type C & $21(42 \%)$ & $20(40 \%)$ \\
Type A & $15(30 \%)$ & $11(22 \%)$ \\
\hline
\end{tabular}

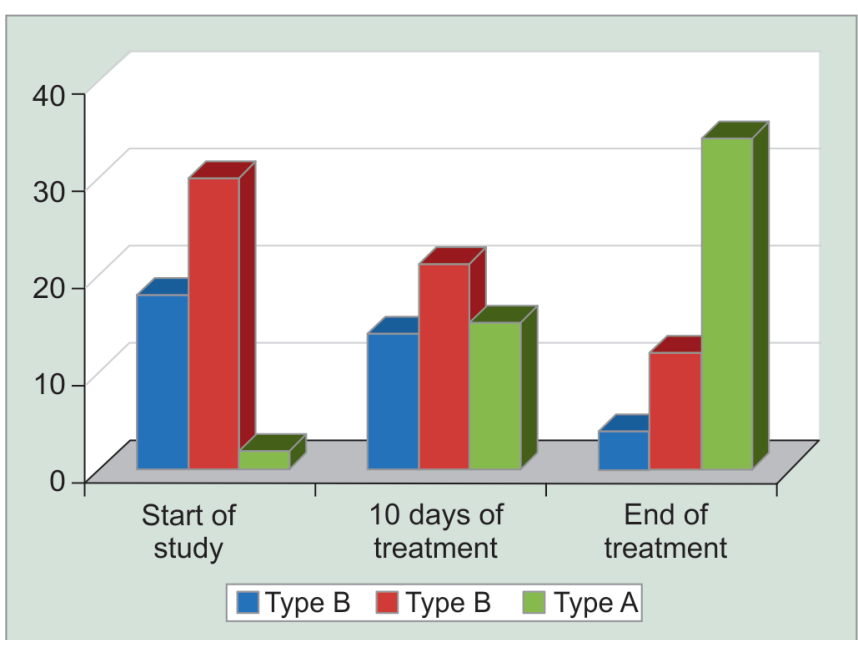

Graph 1: Effect on impedance audiometry at the start of study, 10 days of treatment, and at the end of treatment in group I a total of 18 ears converted to type $\mathrm{A}$ at the end of treatment.

- In group II, 21 ears ( $42 \%$ ) had type B tympanogram at the start of treatment; of which, two ears tympanogram converted into type A at 10 days of treatment and a total of eight ears tympanogram reverted to type A at the end of treatment.

- In group II, 25 ears (50\%) had type C tympanogram; out of which, 5 ears and total of 16 ears tympanogram converted into A type at 10 days and at the end of treatment respectively.

\section{DISCUSSION}

The role of intranasal steroids in the treatment of allergic rhinitis is well established. They are proven to be efficacious and are recommended as first-line therapy for individuals with persistent moderate/severe rhinitis. ${ }^{4}$ Fluticasone propionate is an established intranasal steroid for the treatment of rhinitis, including seasonal allergic rhinitis. Its favorable pharmacological profile and high local efficacy with low systemic bioavailability has established fluticasone propionate as an effective intervention. ${ }^{5}$

Yaman et al ${ }^{6}$ investigated the dose-related effectiveness of corticosteroids in the treatment of OME by using objective measurement techniques of tympanometry

Table 2: Impedance audiometry at the start of study

\begin{tabular}{lll}
\hline Tympanogram & Group I & Group II \\
\hline Type B & $18(36 \%)$ & $21(42 \%)$ \\
Type C & $30(60 \%)$ & $25(50 \%)$ \\
Type A & $02(4 \%)$ & $04(8 \%)$ \\
\hline
\end{tabular}

Table 4: Impedance audiometry 6 weeks after treatment

\begin{tabular}{lll}
\hline Tympanogram & Group I & Group II \\
\hline Type B & $4(8 \%)$ & $13(26 \%)$ \\
Type C & $12(24 \%)$ & $9(18 \%)$ \\
Type A & $34(68 \%)$ & $28(56 \%)$ \\
\hline
\end{tabular}

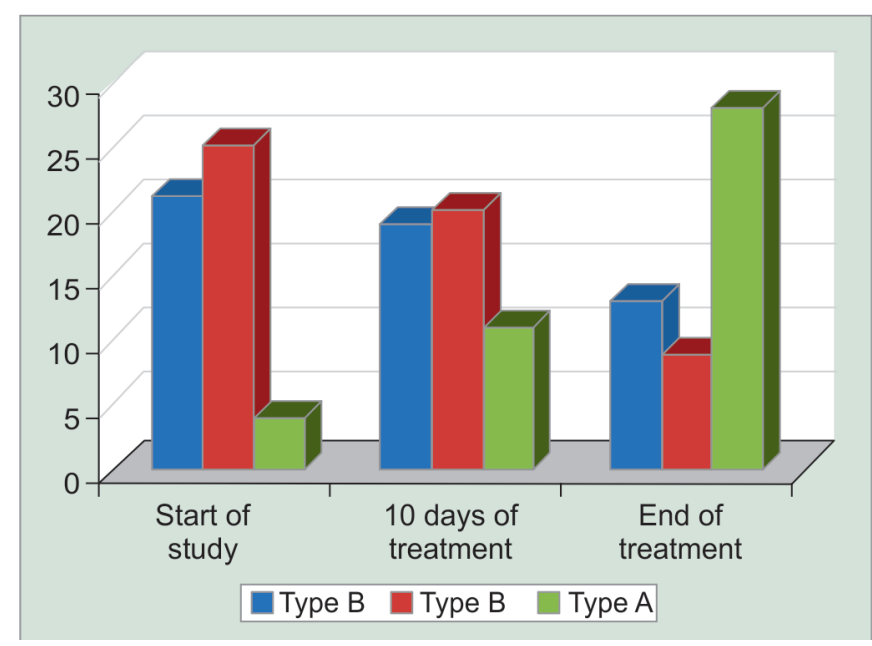

Graph 2: Effect on impedance audiometry at the start of study, 10 days of treatment and at the end of treatment in group II 
and cytokine measurement. The study confirmed the short-term beneficial effect of systemic steroids on the resolution of OME. Seehusen and MacDonnell ${ }^{7}$ conducted randomized controlled trials comparing oral or topical nasal steroids alone or in combination with another agent, such as an oral antibiotic. They concluded that oral steroids, especially when used in combination with an oral antibiotic, lead to quicker resolution of otitis media with effusion in the short-term.

This study demonstrated the outcome of systemic steroids and intranasal steroidal spray in the treatment of middle ear dysfunction on 50 patients aged between 15 and 50 years. In our study, impedance audiometry was done at the start of study and showed type B tympanogram in $36 \%$ ears in group I and $21 \%$ in group II and type C tympanogram in 60\% in group I and 50\% in group II.

Impedance audiometry was repeated at 10 days of treatment and showed reversal to type A curve in $26 \%$ of ears in group I and $14 \%$ of ears in group II. Impedance audiometry at the end of treatment demonstrated reversal to type A tympanogram in $64 \%$ of ears in group I and $48 \%$ of ears in group II.

\section{CONCLUSION}

In our study, it was concluded that:

- Allergic rhinitis causes middle ear dysfunction.

- Corticosteroids have a protective effect on middle ear function.
- Short-term oral steroids, especially when used in combination with intransal steroids, lead to quicker normalization of Eustachian tube dysfunction in comparison with intranasal steroids alone.

- Combination of oral and intranasal steroids appears to have a better effect on Eustachian tube dysfunction in cases of allergic rhinitis.

\section{REFERENCES}

1. Friedman RA, Doyle WJ, Casselbrant ML, Bluestone C, Fireman P. Immunologic mediated eustachian tube obstruction: a double-blind crossover study. J Allergy Clin Immunol 1983 May;71(5):442-447.

2. Martines F, Bentivegna D. Audiological investigation of otitis media in children with atopy. Curr Allergy Asthma Rep 2011 Dec;11(6):513-520.

3. Martines F, Martines E, Sciacca V, Bentivegna D. Otitis media with effusion with or without atopy: audiological findings on primary school children. Am J Otolaryngol 2011 Nov-Dec; 32(6):601-606.

4. Bianchi PG, Agondi R, Stelmach R, Cukier A, Kalil J. Fluticasone furoate nasal spray in the treatment of allergic rhinitis. Ther Clin Risk Manag 2008 Apr;4(2):465-472.

5. Kariyawasam HH, Scadding GK. Seasonal allergic rhinitis: fluticasone propionate and fluticasone furoate therapy evaluated. J Asthma Allergy 2010 Jun 21:3:19-28.

6. Yaman H, Ozturk K, Uyar Y, Gurbilek M. Effectiveness of corticosteroids in otitis media with effusion: an experimental study. J Laryngol Otol 2008 Jan;122(1):25-30.

7. Seehusen DA, MacDonnell J. Steroids for the treatment of otitis media with effusion in children. Am Fam Physician 2012 Feb 1;85(3):235-236. 